$k+2, k+1$, or $k$ such $G$ 's. The number is $k+2$ when there is more than one invariant of each of values 2 and 4 in $H$. When there is only one invariant of one of these values and more than one of the other, the number of $G$ 's is $k+1$. Finally, there are only $k$ such $G$ 's when $H$ contains only one invariant of each of the values 2 and 4 .

It may be added that in the study of all the possible nonabelian groups of order $p^{m}$ which contain an abelian subgroup of order $p^{m-1}$ it is especially desirable to know all of those groups which contain more than one such subgroup, as the other possible groups are distinct whenever they transform the abelian subgroup in different ways. When there is more than one abelian subgroup of order $p^{m-1}$ in $G$, two such subgroups may be transformed differently by the remaining operators.

The UNIVERSITY OF ILLINOIS, September, 1906.

\title{
NOTE ON SYSTEMS OF IN- AND CIRCUMSCRIBED POLYGONS.
}

BY MISS S. F. RICHARDSON.

(Read before the American Mathematical Society, October 27, 1906.)

Is a paper read before the London Mathematical Society on March 12, 1874 (Proceedings of the London Mathematical Society, volume 5) Wolstenholme assumes two similar and similarly situated polygons of $n$ sides, $A B C \ldots K L M$ and $a b c \ldots k l m$, and considers the conditions for an infinity of polygons which shall be inscribed in one of the similar polygons and circumscribed about the other.

He assumes that if $a b$ meet $A M$ in $U$ and if $a m$ meet $A B$ in $V$, then

$$
A U / A M=A V / A B=k .
$$

His solution finds $n-1$ values for $k$, that is, that there are $n-1$ points on $A M$, say, which may be taken as its intersection with $a b$, this point fully determining the polygon $a b c \ldots \mathrm{klm}$.

In particular he finds as the two solutions for the case $n=3$ that $a b$ must divide $A C$ in the ratio $\frac{1}{3}$ or in the ratio 1 . In the first case the triangle abc becomes a point, the common 
point of intersection of the lines drawn through the points of trisection of each side of $A B C$ parallel respectively to the other two sides of the triangle. In the second case $a b c$ is a triangle circumscribing $A B C$, the vertices of $A B C$ being the midpoints of the sides of $a b c$.

For the case $n=4$ his three solutions are that the side $a b$ must divide the side $A D$ in one of the ratios $\frac{1}{4} \cos ^{2} \frac{1}{8} \pi$, $\frac{1}{4} \cos ^{2} \frac{2}{8} \pi, \frac{1}{4} \cos ^{2} \frac{3}{8} \pi$, of which the middle one he says applies only to a parallelogram and reduces the quadrangle abcd to a point while the other two give proper solutions and for squares give the theorem: "If $A B C D$ and $a b c d$ be two concentric squares with their sides parallel and the side of one equal to the sum of the side and diagonal of the other, we can find an infinite number of quadrangles $A^{\prime} B^{\prime} C^{\prime} D^{\prime}$ such that $A^{\prime}, B^{\prime}, C^{\prime}, D^{\prime}$ lie on the sides $A B, B C, C D, D A$, and their sides $A^{\prime} B^{\prime}, B^{\prime} C^{\prime}, C^{\prime} D^{\prime}, D^{\prime} A^{\prime}$ pass through $b, c, d, a$ respectively; or such that $A^{\prime}, B^{\prime}, C^{\prime}, D^{\prime}$ lie on the sides $a b, b c, c d, d a$ and their sides $A^{\prime} B^{\prime}, B^{\prime} C^{\prime}, C^{\prime} D^{\prime}, D^{\prime} A^{\prime}$ pass through $D, A$, $B, C$ respectively."

He adds that in this case and for the parallelogram in general, the in- and circumscribed polygon can never, as far as he has investigated, be a convex polygon.

It will be of interest to compare the properties of the triangle and quadrangle shown by this solution with those properties shown by the synthetic solution of the general problem as proposed by Poncelet of determining the "lieu du sommet libre d'un polygone variable, dont les autres sommets parcourent des droites données, tandis que ses côtés pivotent sur des points fixes," and the "cas pour lesquels le lieu des sommets libres s'abaisse au premier degré."

Taking arbitrarily one of the polygons $A B C \cdots K L M$ and $n-2$ consecutive vertices $b, c, \cdots, k, l$ of the other polygon, the problem of finding the remaining two vertices of the second polygon is the elementary problem of finding two centers $a$ and $m$ from which two projective point rows $A B$ and $L M$ may be projected into the same point row on a given line $A M$. The solution is known. The locus of the vertex $a$ is the line $M M^{\prime}$ joining $M$ to the point on $A B$ which corresponds to $M$ regarded as a point of the point row $L M$, and the locus of $m$ is the line $A A^{\prime}$ joining $A$ to the point on $L M$ which corresponds to $A$ regarded as a point of the point row $A B$, a pair of the required vertices being found as the intersections respectively of $M M^{\prime}$ 
and $A A^{\prime}$ with the join of any third pair of corresponding points on $A B$ and $L M$.

In the case of the triangle $A B C$, the point rows on $A B$ and $B C$ projected from the given vertex $b$ of the triangle $a b c$ being perspective, the vertices $a$ and $c$ must either coincide with $b$ or lie on a line through $B$. That is, the triangle $a b c$ reduces to a point or is circumscribed to $A B C$.*

Hence the necessary and sufficient conditions that there be an indefinite number of triangles inscribed in one of two triangles and circumscribed about the other is that the two triangles be one inscribed in the other, the poles being the vertices of the circumscribed triangle.

Wolstenholme's solution for the triangles is a special case of this solution.

In the case of the quadrilateral $A B C D$ and the given vertices $b$ and $c$ of the quadrilateral $a b c d$, if the point rows on $A B$ and $C D$ projected respectively from the vertices $b$ and $c$ into the point row $B C$ are perspective to each other, then the joins of $A$ and $B$ to their corresponding points on $C D$ and the joins of $C$ and $D$ to their corresponding points on $A B$ must all four meet in some point $P$, and the given vertices $b$ and $c$ must lie on some line through the point $(A B, C D)$. They are respectively the intersections of this line with $C P$ and $B P$. Similarly the vertices $a$ and $d$ must also lie on a line through the point $(A B, C D)$. They may be the intersections of $D P$ and $A P$ with any second line through the point $(A B, C D)$.

It follows that a sufficient condition for a system of in- and circumscribed quadrilaterals with respect to two quadrilaterals is that the two quadrilaterals be in homology, and that a pair of opposite sides of one and a pair of opposite sides of the other be concurrent.

The center of homology $P$, say, might be made the intersection of the diagonals of $A B C D$. And then the points $d$ and $c$ (or $a$ and $b$ ) might chance to be collinear with the point $(C B$, $D A$ ) [assuming that by construction $a$ and $b$ and therefore $b$

* Mr. Cayley, by his solution of the following problem proposed by Mr. W. K. Clifford : Through the angles $A, B, C$, of a plane triangle straight lines $A a, B b, C c$, are drawn. A straight line $A R$ meets $C c$ in $R ; R B$ meets $A a$ in $P ; P c$ cuts $B b$ in $M ; M A$ meets $C c$ in $r$; and so on. Prove that after going twice round the triangle in this way, we always come back to the same point. (Cayley's Collected Math. Papers, volume 5, p. 589) has shown that if the vertices of the inscribed triangle be made the poles there will be closure for all points if the projection be made twice round the triangle. 
and $c$ are collinear with the point $(A B, D C)]$. Then the point rows $A B$ and $D C$ would also be perspective and the points $a$ and $b$ would therefore also be collinear with the point $(C D, D A)$. This case is represented in the diagram below.

Hence, if $A B C D$ be any quadrilateral and if through the point $(B A, C D)$ a line be drawn meeting the diagonals $A C, B D$ in the points $a, b$, respectively, and if these points be joined to the point $(B C, D A)$ by lines meeting the diagonals $B D, A C$ in the

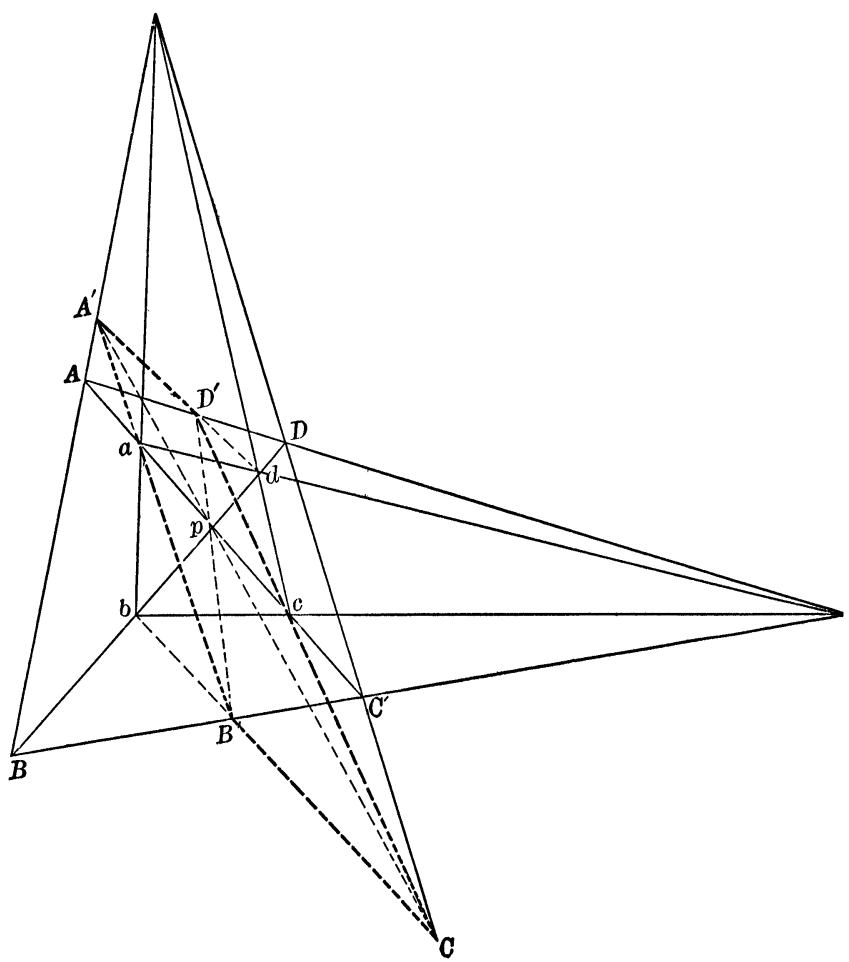

points $d, c$, respectively, then the line dc passes through the point $(B A, D C)$ and there exists an indefinite number of quadrilaterals inscribed in $A B C D$ and circumscribed about abcd if $A B$ be projected on $B C, B C$ on $C D, C D$ on $D A, D A$ on $A B$ from the vertices $c, d, a, b$, respectively, or from the vertices $a, b, c, d$, respectively. It is immaterial which of the two quadrilaterals be named $A B C D$. 
Hence, also, if $A B C D$ and abcd be two concentric parallelograms similar and similarly placed, there exist with respect to them an indefinite number of in-and circumscribed quadrilaterals if $A B$ be projected on $B C, B C$ on $C D, C D$ on $D A, D A$ on $A B$ from the vertices $c, d, a, b$, respectively, or $a, b, c, d$, respectively, or if $a b$ be projected on $b c, b c$ on $c d$, $c d$ on $d a, d a$ on $a b$ from the vertices $C, D, A, B$, respectively, or from $A, B, C$, $D$, respectively.

Moreover in this case the in- and circumscribed quadrilaterals are all parallelograms; for the point rows $A B$ and $C D$ as well as the point rows $A D$ and $B C$ are perspective, the center of projection for both pairs of point rows being the point $P$, the intersection of the diagonals of $A B C D$. Hence the diagonals of each of the variable quadrilaterals pass through $P$, since each diagonal joins a pair of corresponding points of perspective point rows whose center of projection is $P$. Since these diagonals are all bisected at $P$ the variable quadrilaterals are parallelograms.

It follows from this proof that beside the system of in- and circumscribed quadrilaterals obtained by Wolstenholme with respect to the concentric squares referred to above, there is another system obtained by projecting $A B$ on $B C, B C$ on $C D$, $C D$ on $D A, D A$ on $A B$, from the vertices $c, d, a, b$, respectively, or from the vertices $a, b, c, d$, respectively, or by projecting $a b$ on $b c, b c$ on $c d, c d$ on $d a, d a$ on $a b$, from the vertices $C, D, A, B$, respectively, or from the vertices $A, B, C, D$, respectively, and that in this case the variable quadrilaterals are parallelograms instead of reentrant quadrilaterals.

If $A B C D$ be a quadrilateral and if through the point $(A B$, $C D$ ) lines be drawn parallel to $B C$ and $A D$ respectively and through the point $(B C, A D)$ lines parallel to $B A$ and $C D$ respectively, these lines form a quadrilateral in homology with $A B C D$. Hence there is an infinite system of in- and circumscribed quadrilaterals with respect to these two quadrilaterals. The vertices of either may be used as poles.

If $A B C D E F \ldots$ be any polygon of an even number of sides and if $a, b, c$, etc., be respectively arbitrary points on the diagonals $A C, B D, C E$, etc., there will be closure for the points $A$ and $B$ if $A B$ be projected on $B C, B C$ on $C D, C D$ on $D E$, etc., from the poles $a, b$, $c$, etc. respectively. Hence if any third point on $A B$ be projected into itself arbitrarily, the intersections of the projecting lines with $A C, B D, C E$, etc., 
will be the vertices of a polygon with respect to which and $A B C D E F$. . . there is an indefinite number of in- and circumscribed polygons.

If $A B C D E F \ldots$ be any regular polygon of an even number of sides and if $A C$ intersect $F B$ and $B D$ in $a, b$ respectively, $C E$ intersect $B D$ and $D F$ in $c, d$ respectively, etc., then by the properties of the regular polygon there will be closure for the midpoint of $A B$ (and so for all points of $A B$ ) if $A B$ be projected on $B C, B C$ on $C D, C D$ on $D E$, etc., from the poles $a, b, c$, etc., respectively. Also there will be closure for the midpoint of $A B$ and so for all points of $A B$ if $A B$ be projected on $B C, B C$ on $C D$, etc., from the midpoints of $A C, B D, C E$, etc., respectively. If $A B C \ldots$ have an odd number of sides and the projections be made from poles corresponding to those indicated in these two theorems, there will be closure for all points if the projection be made twice round the polygon.

If $A B C D E$ be any pentagon and if the points $(A C, B D)$, $(B D, C E),(D A, E C),(B E, D A),(A C, E B)$ be named $e, a$, $b, c, d$ respectively, then if $A B C D E$ and abcde be regarded as two simple pentagons there will be a poristic system of pentagons if $a b$ be projected on $b c, b c$ on $c d, c d$ on $d e, d e$ on $e a, e a$ on $a b$ from the vertices $B, C, D, E, A$ respectively. This may be proved by testing for closure when the vertices of $A B C D E$ or of abcde are the points projected.

[Professor Morley has pointed out to me that the simple pentagon $A C E B D$ and any one of the variable pentagons constitute the ten-point, ten-line configuration of Desargues's two perspective triangles.]

Vassar College, October, 1906.

\section{HERMITE'S WORKS.}

Oeuvres de Charles Hermite, publiées sous les auspices de l'Académie des Sciences par Emile Picard. Vol. I. Paris, Gauthier-Villars, 1905. 8vo. $x l+498$ pp.

I.

On January 14, 1901, Charles Hermite passed away. A contemporary and zealous disciple of Gauss, Jacobi, and Dirichlet, the friend and generous rival of Cayley, Sylvester, and Brioschi, 